\title{
Ambiente virtual de aprendizaje para la capacitación tipo inducción, sobre contenidos estratégicos y misionales dirigido a colaboradores del Instituto Colombiano de Bienestar Familiar (ICBF)
}

\section{Virtual learning environment for the induction training on mission and strategic content addressed to collaborators from the Instituto Colombiano de Bienestar Familiar (ICBF)}

\author{
Nancy Olarte López ${ }^{1}$ \\ Universidad Militar Nueva Granada, Colombia \\ Jennifer González Castillo² \\ Instituto Colombiano de Bienestar Familiar
}

Recibido: 10-03-2017

Aceptado: 16-06-2017

\section{Cita RecomendadA}

Olarte, N. y González, J. (2017) Ambiente Virtual de Aprendizaje para la Capacitación Tipo Inducción, Sobre Contenidos Estratégicos y Misionales Dirigido a Colaboradores del Instituto Colombiano de Bienestar Familiar - ICBF. Hamut'ay, 4 (1), 45-59. Recuperado de: http://dx.doi. org/10.21503/hamu.v4i1.1400

\section{RESUMEN}

Se desarrolló un Ambiente Virtual de Aprendizaje (AVA) para la capacitación tipo Inducción a los colaboradores ICBF, mediante contenidos estratégicos y misionales a través de la implementación de diferentes herramientas multimedia para la apropiación de conocimientos que anteriormente sólo se transmitían de forma presencial en las diferentes regionales del ICBF.

El diseño de un AVA mediante espacios Web 2.0 involucró la participación de colaboradores del Instituto. Estos espacios permitieron escenarios colaborativos para gestionar conocimiento e información relevante hacia la capacitación de procesos donde se involucran poblaciones vulnerables de niños, niñas y adolescentes, reduciendo de manera significativa la inversión de recursos económicos logísticos en desplazamiento, capital humano y tiempos invertidos en capacitaciones en sitios presenciales. Asimismo, el AVA, como instrumento para la capacitación tipo inducción, aportó ampliamente al fortalecimiento del desempeño y competencias de los colaboradores ICBF como entidad pública, desde un modelo cooperativo.

En primer lugar, se lleva a cabo una descripción detallada del modelo de aprendizaje apropiado al AVA, introducción al ICBF desarrollado, luego se indican las fases del diseño del AVA, junto con el Objeto Virtual de Aprendizaje - OVA - Misional Estratégico. Por otro lado, se indica detalladamente la arquitectura del diseño y la construcción del AVA. En seguida se describen las herramientas tenidas en cuenta para las pruebas hacia los colaboradores ICBF, junto con sus resultados. Mediante

1 Docente de Planta Universidad Militar Nueva Granada, Magister en Tecnologías de la Información Aplicadas a la Educación - ETIAE Universidad Pedagógica Nacional - UPN, Especialista en Tecnologías de la Información Aplicadas a la Educación - ETIAE Universidad Pedagógica Nacional - UPN Ingeniero en Telecomunicaciones Universidad Militar Nueva Granada, nancy.olarte@unimilitar.edu.co. 2 Integradora Virtual Administradora Escuela Instituto Colombiano de Bienestar Familiar - ICBF, Especialista en Tecnologías de la Información Aplicadas a la Educación - ETIAE Universidad Pedagógica Nacional - UPN, Ingeniero de Sistemas Universidad Distrital Francisco José de Caldas, Jennifer.GonzalezC@icbf.gov.co. 
estrategias tecnológicas basadas en una capacitación tipo inducción se formó a colaboradores en lo estratégico y misional del instituto, incursionando de una manera semipresencial, con acogida de la herramienta diseñada.

Palabras Clave: AVA, capacitación, contenido, estratégico, inducción, misional, tecnologías.

\section{Abstract}

A Virtual Learning Environment (VLE) was developed as part of an induction training addressed to $I C B F$ collaborators. It combined strategic and mission content through the implementation of different multimedia tools, provided that it facilitates the appropriation of knowledge that was previously transmitted only through face- to- face interaction in the different regions of the ICBF. The design of an VLE through Web 2.0 spaces, involved the participation of collaborators from the Institute. These spaces allowed collaborative scenarios to manage knowledge and information relevant to the training, in processes involving vulnerable population of children and adolescents, significantly reducing in this way, the investment of economic resources in logistics, travel expenses, human capital and time invested in on-site training. In the same way, the VLE, as an instrument for induction training, greatly contributed to the strengthening of the practices and competences of the $I C B F$ collaborators as a public entity, deriving out of a cooperative model.

Firstly, we deal with a detailed description of the learning model pertinent to the VLE, an explained introduction to the $I C B F$, then we state the phases of the VLE design, along with the Virtual Learning Object - VLO - Strategic and Mission related content. On the other hand, the architecture of the VLE design and its construction is carefully detailed. Next, the tools used to test the ICBF collaborators are described, along with their results. Through technological strategies based on an induction training, collaborators were trained in relation to strategic and mission content of the institute, accessing in a blended way, with a pleasing acceptance of the designed tool.

Keywords: VLE, training, content, strategic, induction, mission, technologies

\section{INTRODUCCIÓN}

Desarrollo del Ambiente Virtual de Aprendizaje - AVA

El Instituto Colombiano de Bienestar Familiar ICBF, en la actualidad brinda de manera presencial y de cubrimiento parcial, inducción a todos sus colaboradores que hacen parte de una amplia diversidad de población, entre ellos, los vinculados como servidores públicos, contratistas, pasantes, madres comunitarias y profesionales que trabajan día a día para cumplir la misión del instituto que, en otras palabras, es fomentar el desarrollo y la protección integral de la primera infan- cia, la niñez, la adolescencia y el bienestar de las familias de nuestro país.

Llevar a cabo la inducción es un requisito necesario para facilitar la identificación de los colaboradores con la institución (ICBF, 2013). No obstante, capacitarlos en su totalidad se convierte en un reto, ya que se debe considerar que el alcance del instituto es a nivel nacional; es decir, está dirigido hacia 33 regionales y alrededor de 206 centros zonales que hasta el día de hoy se tienen; el trabajo se realiza de manera conjunta en la construcción de una sociedad próspera y equitativa a partir del desarrollo integral de las nuevas generaciones y sus familias (ICBF, 2014). 
Hoy en día, el cubrimiento en capacitación no se logra en su totalidad; por un lado existen zonas apartadas de nuestro territorio nacional con acceso limitado, en las cuales se evidencia dificultad en el desplazamiento del personal y que representa costos logísticos altos para la institución en cuanto a traslados entre y hacia diferentes lugares; por otro, se evidencia el incremento en el número de colaboradores del instituto $\mathrm{y}$, por último, el número de capacitadores con los que cuenta no es suficiente. Aunque se pretende completar el tipo de inducción hacia cada uno de ellos, al ser ésta una tarea ambiciosa pero gratificante, se puede llegar a afirmar que una de las maneras para alcanzar este propósito podría ser mediante la ayuda de las Tecnologías de la Información y Comunicaciones (TIC).

Por otro lado, el instituto tiene sus orígenes en 1968. A partir de allí y, a través de más de cuatro décadas, ha brindado atención a niños y niñas, adolescentes y familias. De manera especial, a aquellos en condiciones de amenaza, insolvencia o vulneración de sus derechos (ICBF, 2011). Uno de los aspectos fuertes a destacar dentro de los recursos de protección ha sido acoger a la población víctima de la violencia, población que al ser analizada aumentó de 23 a 82 por cada 100.000 habitantes durante los años 1980 a 1985 . Esta cifra angustiante evidenciada a causa de tendencias como, por ejemplo, el predominio de la fuerza para consecución de fines, tanto en los espacios de la vida privada como en los de la vida pública, e igualmente en las interacciones políticas y en las familiares, y aún en las laborales (ICBF, 2013).

Al retomar el tema de la inducción, surge una pregunta: ¿Capacitar a los colaboradores ICBF mediante las tecnologías de la información en lo misional del instituto, tendrá la misma relevancia que en la forma presencial? Rebatir esta pregunta lleva a comprender hasta el momento dos variables a abarcar: Funcionalidad y Suficiencia.

Para comenzar, algo es "funcional" si logra satisfacer las necesidades de sus usuarios. Al relacionar este término hacia el aspecto de una capacitación (variable dependiente), de manera particular, se tienen dos tipos de usuarios: los iniciales colaboradores ICBF -quienes recibirán la inducción misional con fines estratégicos para el desarrollo y fortalecimiento de la capacidad organizacional del instituto-y la población vulnerable, quienes recibirán el apoyo y atención de los primeros. Pensar ante todo en garantizar una excelente inducción llevaría a los colaboradores no sólo a tener alto nivel de pertenencia al instituto, o potenciar su excelencia y desarrollo humano, sino también un incremento en el desempeño de sus funciones, al satisfacer las necesidades de los segundos.

Por otro lado, se piensa en "suficiencia", es necesario plantear si las tecnologías de la información serán adecuadas como apoyo a una óptima capacitación, inclusive si a través de varias décadas se ha manejado por modalidad presencial. Reflexionar en este paradigma permite relacionar factores como la capacidad informática y ofimática de las regionales y si sus colaboradores tienen o no conocimiento en su manejo; además de aspectos como su ubicación geográfica dentro del territorio nacional, que en ocasiones es limitante para el alcance tecnológico.

Pero, si se tuviesen las condiciones ideales o pertinentes, sería más que significativo cumplir con el objeto de capacitar a todos los funcionarios, incluso en términos de optimización de las funciones de la entidad, más aún cuando de por medio se tengan las herramientas de información y tecnología necesarios. No obstante, se debe resaltar que el trabajo colaborativo que ha tenido relevancia a nivel presencial puede tener una transición a su vez, al ser de manera interactiva. No sólo el hecho de aprender nuevas herramientas e interactuar con ellas aumenta la curiosidad, sino que la motivación al estar frente a aplicaciones atractivas permite incrementar el conocimiento y satisfacción personal.

Sin embargo, no se pretende que el acceso por parte de la población a las TIC y el desarrollo social del país ocasionen un distanciamiento, sino que, al contrario, vayan soportadas una con la otra, de manera cooperativa. De forma singular, en el caso del ICBF, no sólo se pretende informar, comprender y fomentar la actualidad tecnológica, sino también que, a través de un ambiente colaborativo y accesible, se lleve a cabo procesos de capacitación en inducción. 
Ambiente Virtual de Aprendizaje para la Capacitación Tipo Inducción, Sobre Contenidos Estratégicos y Misionales Dirigido a Colaboradores del Instituto Colombiano de Bienestar Familiar (ICBF)

\section{Diseño y desarrollo del Ambiente Virtual de Aprendizaje - AVA}

En primer lugar, se ideó que el AVA (variable independiente) estuviera conformada por módulos didácticos, uno de generalidades del ICBF y el otro misional, ambos diseńados para abordar procesos de formación permanente que se implementan actualmente en el instituto (ver figura 1), desde los cuales el sentido de atención hacia los colaboradores sea mejorado (Medina, 2016) a través de los contenidos, recursos y actividades a implementar; además de que a tiempo futuro se incremente el número de los módulos de este ambiente inicial.

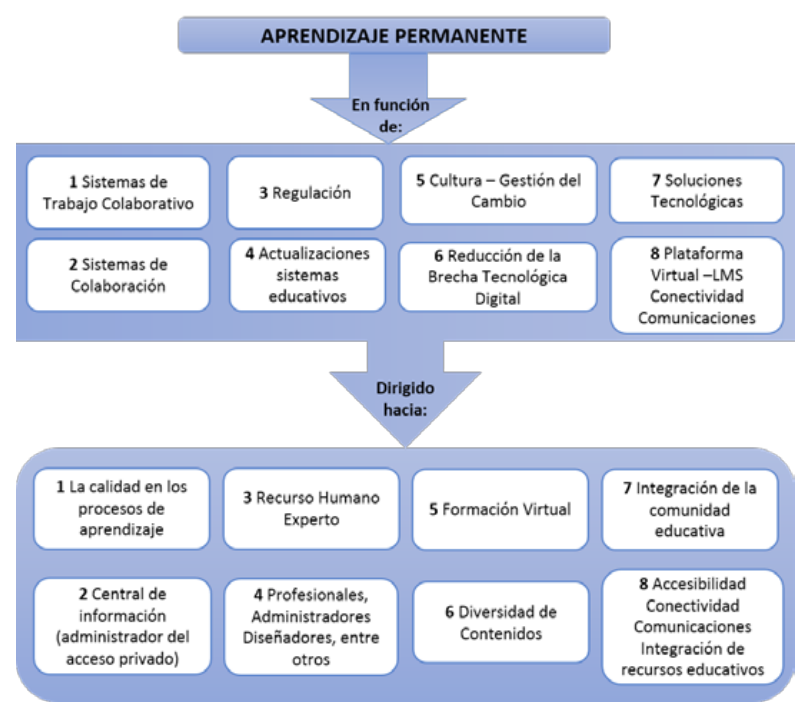

Figura 1. Situación deseada: aprendizaje permanente en el ICBF.

Fuente: Elaboración propia.

Luego de que el estudiante navegue por los contenidos multimedia y lleve a cabo actividades de aprendizaje y evaluaciones de ambos módulos, al cumplir con los requisitos establecidos podrá descargar, de manera automática, el certificado de la Escuela ICBF.

Asimismo, dentro de los aspectos operativos para el levantamiento de información inicial, se llevaron a cabo entrevistas a 10 colaboradores aleatorios, identificados como líderes de cada área de los procesos institucionales y programas del Instituto.

Por otro lado, dentro del diseño del AVA, se tuvieron en cuenta las fases cuyas actividades se describen en la tabla 1.
Tabla 1

Fases del proyecto

\begin{tabular}{|c|c|}
\hline Fase & Actividad \\
\hline \multirow[t]{2}{*}{$\begin{array}{l}\text { Levantamiento } \\
\text { de información }\end{array}$} & $\begin{array}{l}\text { Selección y definición de los contenidos por } \\
\text { cada una de las temáticas a desarrollar. }\end{array}$ \\
\hline & Establecimiento del cronograma de actividades. \\
\hline \multirow[t]{9}{*}{ Análisis } & Revisión de materiales entregados \\
\hline & Definición de la población objetivo. \\
\hline & $\begin{array}{l}\text { Descripción de temáticas de los objetos de } \\
\text { aprendizaje. }\end{array}$ \\
\hline & Definición de modelo pedagógico. \\
\hline & $\begin{array}{l}\text { Definición de objetivos de aprendizaje y alcance } \\
\text { temático del curso. }\end{array}$ \\
\hline & Definición de la estructura general del curso. \\
\hline & $\begin{array}{l}\text { Escritura de contenidos, diseño de actividades } \\
\text { de aprendizaje, evaluaciones y selección de } \\
\text { contenidos de estudio. }\end{array}$ \\
\hline & Módulo: Eje Institucional. \\
\hline & Módulo: Eje Misional \\
\hline \multirow[t]{5}{*}{ Diseño } & Construcción y revisión de maqueta (Prototipo). \\
\hline & Definición de línea gráfica. \\
\hline & $\begin{array}{l}\text { Propuesta de diagramación de los objetos } \\
\text { virtuales de aprendizaje. }\end{array}$ \\
\hline & Elaboración del manual de usuario. \\
\hline & Alistamiento del ambiente virtual de aprendizaje. \\
\hline Desarrollo & $\begin{array}{l}\text { Producción de los módulos eje institucional, eje } \\
\text { misional. }\end{array}$ \\
\hline \multirow[t]{4}{*}{ Implementación } & Montaje en plataforma de la Escuela ICBF. \\
\hline & Pruebas técnicas de los objetos de aprendizaje. \\
\hline & Diseño de manual de usuario. \\
\hline & Prueba piloto con colaboradores del ICBF. \\
\hline
\end{tabular}

Fuente: Elaboración propia.

Por otro lado, cabe resaltar que dentro de la estructura del desarrollo del AVA no solo era necesario tener un diseño instruccional, sino que era indispensable contar con un modelo pedagógico. El ICBF, dentro de sus capacitaciones institucionales siempre ha implementado el cognitivismo, el mismo que fue también implementado al AVA. Este modelo cuenta con características como: (i) énfasis en el conocimiento significativo, (ii) la participación activa del estudiante en el proceso de aprendizaje, (iii) creación de ambientes de aprendizaje que permitan y estimulen a los estudiantes a hacer conexiones mentales con material previamente aprendido, (iv) la estructuración, organización y secuencia de la información para facilitar su óptimo procesamiento (Casas, 2016). 
Asimismo, este modelo se encuentra basado en procesos de razonamiento y asimilación de información que todo individuo tiene durante su vida, sea durante su proceso educativo dentro de la escuela o fuera del entorno escolar, a temprana edad o en educación superior (Beltrán, 2016).

Debido al énfasis en las estructuras mentales, se considera a las teorías cognitivas más apropiadas para explicar las formas complejas de aprendizaje (razonamiento, solución de problemas, procesamiento de información).

Por otro lado, en cuanto a la integración de contenidos, mediante la siguiente gráfica (figura 2) se describen las teorías que se aplicarán mediante las Tecnologías de la Información y Comunicación (TIC), en el AVA de Inducción al ICBF:

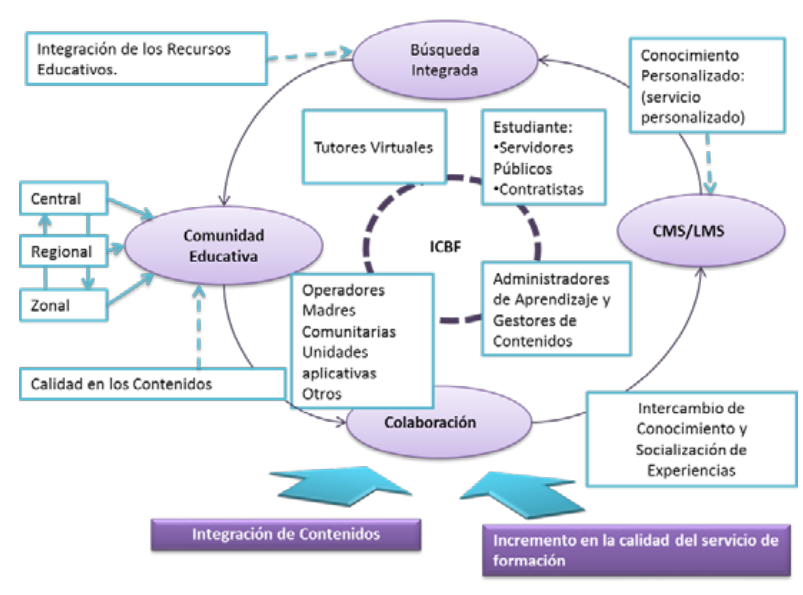

Figura 2. Integración contenidos ICBF.

Fuente: ICBF.

A partir de estas condiciones, el curso de Inducción al ICBF utilizó la plataforma virtual de la Universidad Pedagógica Nacional - UPN, llamada Kenta, la cual permitió que no sólo se subieran los contenidos, sino también que se desarrollaran varias pruebas, como, por ejemplo: de navegabilidad entre la población estudio y su posterior descripción detallada. La tabla 2 describe las restricciones o pseudorequerimientos a tener en cuenta a nivel tecnológico para el óptimo desarrollo y navegación del AVA dentro de la plataforma Kenta.
Tabla 2

Pseudorequerimientos

\begin{tabular}{|c|c|}
\hline Identificador & Características \\
\hline RN1: Red - Internet & $\begin{array}{l}\text { Computadores con conexión a internet. } \\
\text { Desde y hacia los recursos propios del } \\
\text { ICBF. }\end{array}$ \\
\hline RN2: Plataforma & LMS Moodle. \\
\hline Tecnológica & $\begin{array}{l}\text { Identificación de usuarios de estudiantes } \\
\text { con sus respectivas contraseñas (con } \\
\text { parámetros de seguridad con políticas } \\
\text { de acceso local y remoto) }\end{array}$ \\
\hline $\begin{array}{l}\text { RN3: Sistema } \\
\text { Operativo }\end{array}$ & Preferiblemente Windows XP - 8 . \\
\hline RN4: Navegador Web & $\begin{array}{l}\text { Preferiblemente Chrome deshabilitando } \\
\text { elementos emergentes. }\end{array}$ \\
\hline RN5: Software & $\begin{array}{l}\text { De tipo aplicación hacia usuario final } \\
\text { como Flash y sus complementos (Ver- } \\
\text { sión CS3 o superior). }\end{array}$ \\
\hline $\begin{array}{l}\text { RN6: Tamaño de } \\
\text { archivos }\end{array}$ & $\begin{array}{l}\text { Entre } 7 \mathrm{MB} \text { y } 15 \mathrm{MB} \text {, dependiendo de la } \\
\text { actividad propuesta por el docente. }\end{array}$ \\
\hline
\end{tabular}

Fuente: Elaboración propia.

Dentro de las estrategias para cada meta de aprendizaje y contenido se pueden tener varias estrategias pedagógicas. Cada una de estas ha descrito los criterios a seguir (tabla 3).

Tabla 3

Estrategias pedagógicas

\begin{tabular}{ll}
\hline Meta de & Al ingresar el colaborador a la institución, se le \\
Aprendizaje & $\begin{array}{l}\text { aportará conocimientos e información básica de } \\
\text { la entidad, creándose pertenencia en procesos y } \\
\text { actividades misionales. }\end{array}$ \\
\hline Contenido & Indicación del contenido específico. \\
& - Módulo \\
& - Introducción \\
& - Objetivos estratégicos. \\
& - Contexto histórico. \\
& - Misión y visión. \\
& - Principios institucionales. \\
& - Malores institucionales. \\
& - Estructura organizacional. \\
\hline Sstrategia & Se realiza una estrategia: \\
& - Fase de motivación: \\
- Fase de comprensión \\
- Fase de adquisición. \\
- Fase de retención. \\
- Fase de recuerdo. \\
- Fase de generalización. \\
- Fase de ejecución.
\end{tabular}


Ambiente Virtual de Aprendizaje para la Capacitación Tipo Inducción, Sobre Contenidos Estratégicos y Misionales Dirigido a Colaboradores del Instituto Colombiano de Bienestar Familiar (ICBF)

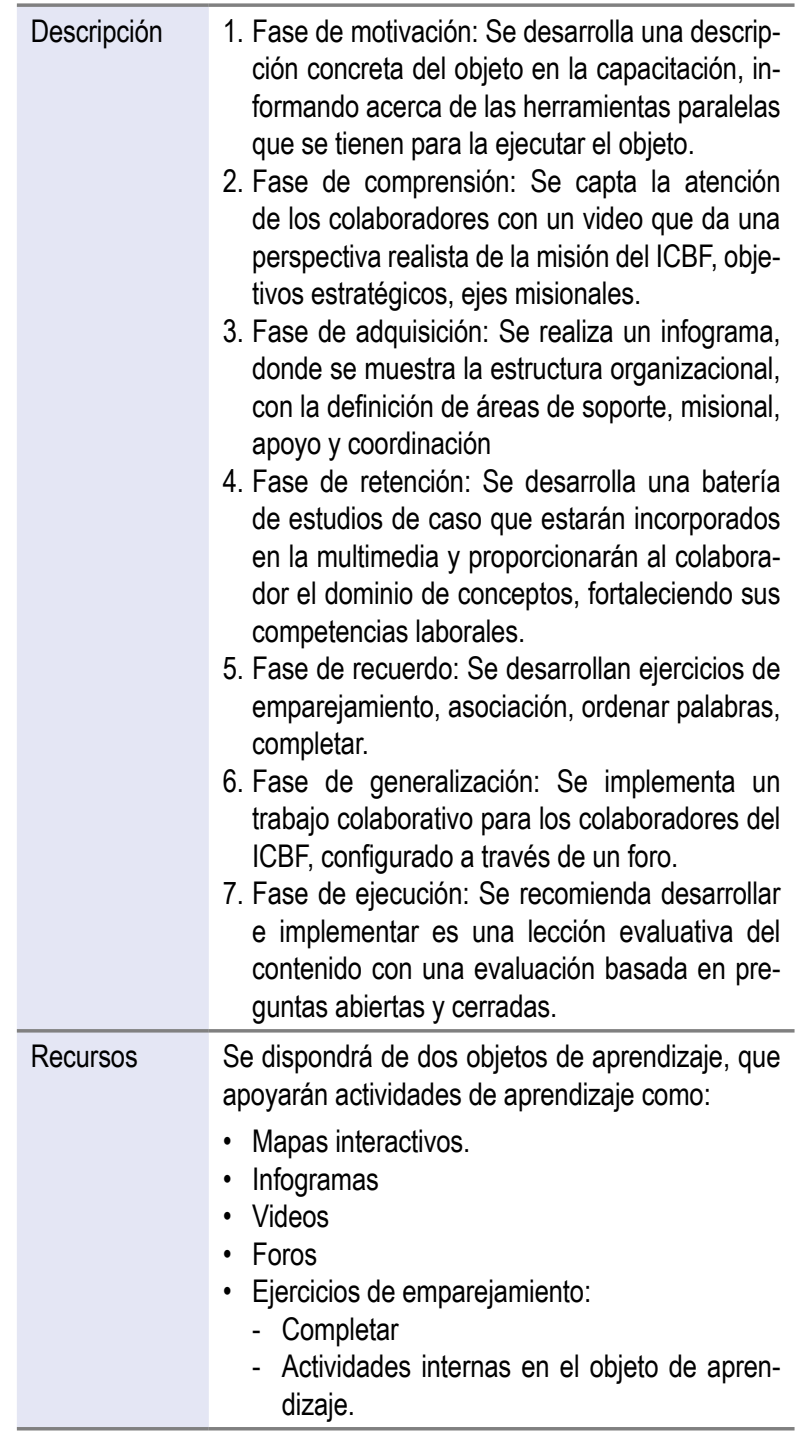

Fuente: Elaboración propia.

En la tabla 4 se describe los contenidos del AVA involucrados.

Tabla 4

Contenidos involucrados en el ambiente

\begin{tabular}{|c|c|c|}
\hline Contenido & Descripción & Metodología \\
\hline $\begin{array}{l}\text { Foro de } \\
\text { Presentación }\end{array}$ & $\begin{array}{l}\text { Diseñado para } \\
\text { el colaborador } \\
\text { del ICBF. }\end{array}$ & $\begin{array}{l}\text { El colaborador realiza una breve } \\
\text { presentación (nombres, apelli- } \\
\text { dos, profesión, cargo, ciudad, ex- } \\
\text { periencia las expectativas frente } \\
\text { al Curso). }\end{array}$ \\
\hline \multirow{3}{*}{$\begin{array}{l}\text { Fase ¿Qué } \\
\text { tanto } \\
\text { sabemos? }\end{array}$} & \multirow{3}{*}{$\begin{array}{l}\text { Se busca que } \\
\text { el participante } \\
\text { identifique los } \\
\text { conocimientos } \\
\text { previos frente } \\
\text { a las temá- } \\
\text { ticas que se } \\
\text { abordarán en el } \\
\text { módulo. }\end{array}$} & ACTIVIDAD 1 \\
\hline & & $\begin{array}{l}\text { Conocer los conceptos previos } \\
\text { que tienen los participantes } \\
\text { frente al Instituto Colombiano de } \\
\text { Bienestar Familiar. }\end{array}$ \\
\hline & & $\begin{array}{l}\text { Metodología: Los participantes } \\
\text { deberán responder la pregunta } \\
\text { del foro: ¿Qué es el Bienestar } \\
\text { Familiar para ti? }\end{array}$ \\
\hline
\end{tabular}

Criterios de evaluación: Esta actividad no será evaluada; se utiliza como diagnóstico y es requisito para generar el certificado al final del recorrido multimedia.

\begin{tabular}{ll}
\hline $\begin{array}{l}\text { Fase ¿Qué } \\
\text { debemos }\end{array}$ & Plantea la infor- \\
saber? & mación y fun- \\
& damentación \\
& conceptual, \\
& metodológica \\
& y normativa \\
& que se requiere \\
& para ser parte \\
& del ICBF.
\end{tabular}

ACTIVIDAD 2

Conocer o el Instituto Colombiano de Bienestar Familiar.

Propósito: Conocer las generalidades de ICBF.

Metodología: El participante observará el contenido del video Somos Instituto colombiano de Bienestar Familiar, disponible en https://www.youtube.com/watch?v=MSJBX6Uxh31

ACTIVIDAD 3

Conociendo las generalidades del Instituto colombiano de Bienestar Familiar

Propósito: Conocer las generalidades del Instituto colombiano de Bienestar Familiar por parte de los participantes.

Metodología: El participante observará y navegará por el OVA Estratégico - Misional que explica los antecedentes, misión, visión, objetivos estratégicos, líneas estratégicas, fases, etcétera.

\begin{tabular}{lll}
\hline $\begin{array}{l}\text { Fase ¿Qué } \\
\text { hemos }\end{array}$ & $\begin{array}{l}\text { Los participan- } \\
\text { tes pondrán }\end{array}$ & ACTIVIDAD 4 \\
aprendido? & $\begin{array}{l}\text { a prueba sus tanto sabes del Instituto } \\
\text { conocimientos } \\
\text { adquiridos en el } \\
\text { módulo }\end{array}$ & $\begin{array}{l}\text { Colombiano de Bienestar Fami- } \\
\text { liar? }\end{array}$ \\
& $\begin{array}{l}\text { Propósito: Identificar la apropia- } \\
\text { ción de las generalidades del } \\
\text { instituto colombiano de Bienestar } \\
\text { Familiar a través de una evalua- } \\
\text { ción. }\end{array}$
\end{tabular}

Metodología: El participante deberá realizar la evaluación final y contara con 3 intentos.

Criterios de evaluación: Preguntas para la evaluación final (la evaluación contará con 10 preguntas aleatorias de una batería de 50). De estas se deben responder correctamente como mínimo 8 de 10 preguntas.

Porcentaje de Evaluación: 0-100, donde 100 es la nota más alta y 60 es el mínimo aprobatorio.

\begin{tabular}{ll}
\hline Fase ¿Qué & Se espera que \\
Propongo? & los participan- \\
& tes puedan \\
& proponer las \\
& estrategias o \\
& las acciones
\end{tabular}

Actividad 5

EVALUACIÓN

Propósito: Hacer una aproximación general al Instituto Colombiano de Bienestar Familiar, de sus propósitos, estrategias, principios institucionales, etcétera. 
Metodología: Diseñe un slogan para el instituto Colombiano de Bienestar Familiar teniendo en cuenta los contenidos vistos en este.

Criterios de evaluación: Esta actividad será evaluada a partir del nivel de apropiación conceptual y metodológica del participante frente a las generalidades del ICBF, el cual tendrá la opción de realizar el slogan durante la permanencia en el Módulo.

Fuente: Elaboración propia.

Las dinámicas de interacción que se pueden llevar a cabo entre estudiantes, estudiante-maestro y la navegación del ambiente, se diagraman mediante la figura 3 .

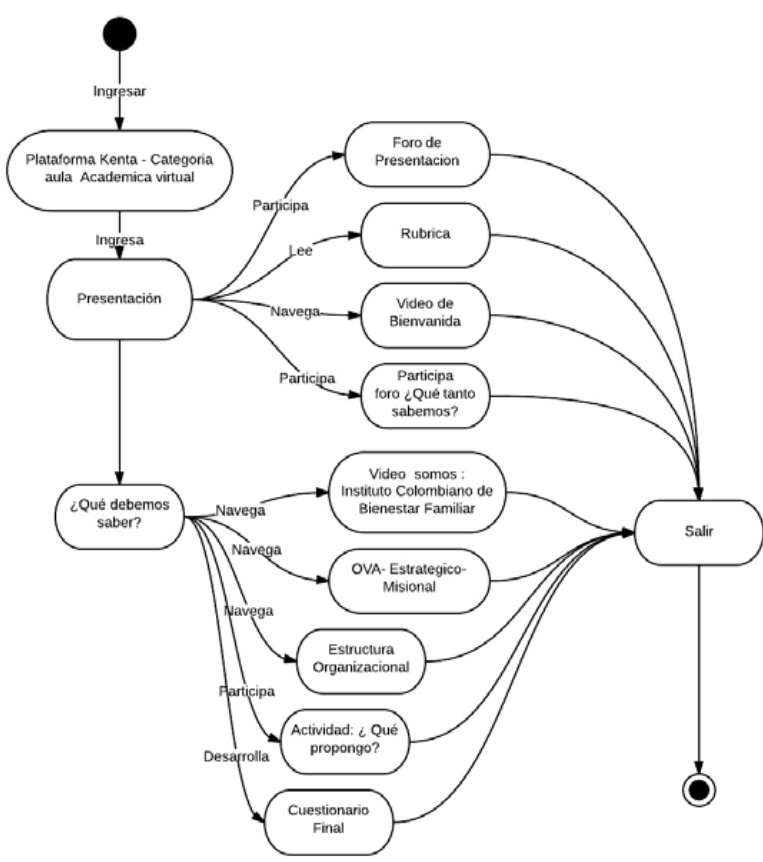

Figura 3. Modelo dinámico navegación AVA. Fuente: Elaboración propia.

Además, dentro de la construcción del Ambiente Virtual de Aprendizaje, la Inducción al ICBF está diseñada para ser un complemento de la capacitación presencial, que se lleva a cabo en cada una de las regionales y centros zonales del ICBF a nivel nacional, hacia los colaboradores ICBF.

Su implementación, incluye también un Objeto Virtual de Aprendizaje, el cual incluye contenidos estratégicos y misionales del instituto, que contribuirán a la formación y compromiso en el tiempo de los nuevos colaboradores ICBF.

Para la estructura del AVA fueron diseñados diferentes espacios de formación y navegación en leguaje HTML, bajo la herramienta Macromedia Dreamveawer CS6. Por otro lado, los botones y algunos escenarios fueron llevados a cabo de manera personalizada, dibujados a mano y luego tratados de forma especial bajo el programa Macromedia Flash.

Para el diseño se tuvieron en cuenta los colores institucionales del ICBF, también conceptos como la usabilidad y la aplicación del uso de color a través de pruebas realizadas según los tipos de aprendizaje e inteligencias múltiples que tienen los colaboradores valorados. Desde el principio se buscó aplicar un sentido motivacional del AVA, con un toque personalizado, que emergió de esquemas verticales o rígidos para la navegación de todos los recursos.

En la figura 4 se tiene la pantalla de inicio del AVA: Inducción al ICBF.

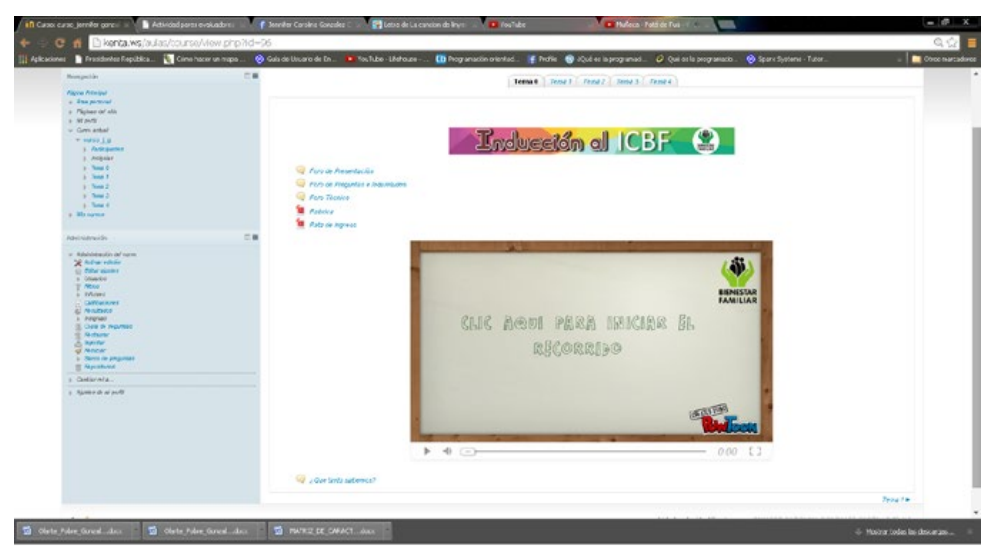

Figura 4. Pantalla de inicio AVA.

Fuente: Elaboración propia.

Por otro lado, en la pantalla de inicio del OVA Estratégico - Misional se tienen los elementos que se muestran en la figura 5 . 

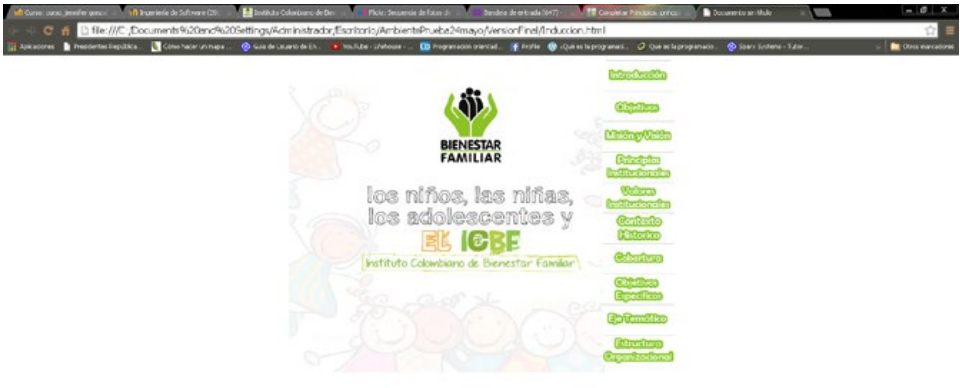

Figura 5. Pantalla de inicio OVA. Fuente: Elaboración propia.

\section{Materiales y Métodos}

\section{Participantes}

El grupo piloto evaluado estuvo conformado por seis (6) hombres y cuatro (4) mujeres, para un total de 10 colaboradores elegidos en forma aleatoria. Estos recibieron una capacitación de inducción en lo misional y estratégico. Sus edades oscilaban entre los 25 y 45 ańos. Cada uno de los sujetos eran servidores públicos que se desempeñaban como contratistas, operadores del sistema, pasantes, madre comunitaria y o profesionales que formaban parte de la misión del ICBF en la ciudad de Bogotá. Pertenecían a niveles socioeconómicos ubicados entre los estratos 2 y 4.

Cada uno de estos colaboradores tuvo previamente una inducción en el modelo basado en poblaciones beneficiadas, incluyendo primera infancia, niñez y adolescencia, familia y comunidad con protección integral y coordinación regional. A su vez, tuvieron una capacitación en valores institucionales, como don de servicio, sentido de pertenencia, innovación, tolerancia, credibilidad, enfoque a resultados, amabilidad y honestidad.

Cabe resaltar que las pruebas realizadas tenían como propósito caracterizar el tipo de aprendizaje de la población de estudio y, ya a nivel del curso, verificar pruebas de navegabilidad.

\section{Instrumentos}

Los instrumentos que se describen a continuación fueron aplicados a colaboradores del ICBF entre enero a mayo de 2014. Fueron de gran ayuda para el desarrollo y valoración tanto de AVA en Inducción al ICBF, como del OVA Estratégico - Misional. Los resultados que se presentan mediante tablas y gráficos se explican en la sección siguiente de este artículo.

Cada instrumento tiene un objetivo específico.

Test de KOLB. El objetivo es caracterizar los tipos de aprendizaje predominantes y luego identificar si las decisiones de color basadas en las inteligencias múltiples fueron o no acertadas. Éste test, cuenta con un total de 9 preguntas, cada una de ellas ofrece 4 opciones de respuesta, a las cuales se les debe asignar un puntaje (en el rango 4-1), considerando que el cuatro (4) es lo que mejor describe al individuo y uno (1) lo que peor lo describe. Este test se realizó en formato Excel y fue aplicado desde 1985.

Encuesta 1 de satisfacción del curso. Su objetivo es identificar si los objetivos del curso se ajustan a los contenidos estratégicos y misionales del ICBF. Consta de 24 preguntas de elaboración propia, enfocadas hacia temas como el desarrollo de contenido, evaluación del curso, respecto a contenidos actividades y evaluación e interacción gráfica. Se realizó mediante la herramienta formularios de Google.

Encuesta 2 Evaluación del ambiente virtual de aprendizaje. En este instrumento se incluyeron 26 preguntas de elaboración propia, distribuidas en cuatro dimensiones: relevancia, pensamiento reflexivo, interactividad, apoyo del tutor, apoyo de compañeros e interpretación. Se realizó mediante la herramienta formularios de Google.

Los tres instrumentos para la valoración del AVA en sus diferentes fases fueron aplicados a colaboradores del ICBF, específicamente en la Sede Dirección General ubicada en la ciudad de Bogotá (Av. Cra. 68 No. 64C-75), Barrio Bosque Popular de la localidad de Engativá (figura 6). 


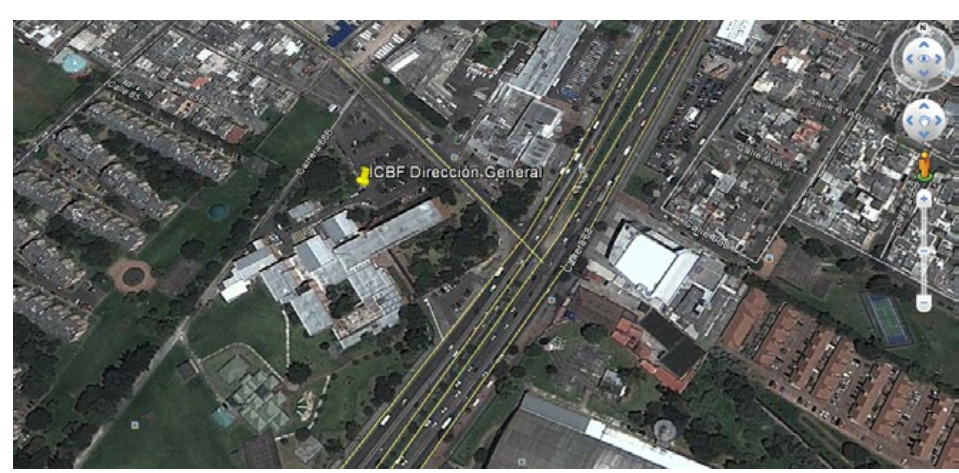

Figura 6. Sede Dirección General ICBF. Fuente: Herramienta Google Earth.

\section{Tipo y diseño}

Aunque se ha realizado un análisis cualitativo de la información, debido a la implementación de un modelo pedagógico cognitivista en los diferentes momentos del AVA, también se obtuvieron resultados cuantitativos, pues se valoraron 10 individuos colaboradores del ICBF, para caracterizar su estilo de aprendizaje, además de su valoración hacia la navegabilidad del curso desarrollado.

Se trata de una investigación no experimental de tipo exploratoria que, además, buscó establecer la confiabilidad del AVA hacia colaboradores ICBF, identificar el estilo de aprendizaje predominante entre los encuestados y las particularidades de favorabilidad o desacuerdo con los contenidos implementados bajo mediación de TIC en el AVA.

El estudio tuvo un alcance exploratorio para conocer la importancia de la capacitación tipo inducción, realizada en forma virtual a colaboradores ICBF. El diseño de investigación es no experimental transeccional, pues las mediciones se realizaron en un solo momento, entre enero a mayo de 2014.

\section{Variables:}

- Variable independiente: Ambiente Virtual de Aprendizaje.

- Variable dependiente: Capacitación tipo inducción.

\section{Resultados}

En este acápite se detallan los resultados de los 3 primeros instrumentos, ya que el último se administró de manera personal a cada colaborador y su grado de subjetividad depende por un lado del manejo eficiente de herramientas informáticas y ofimáticas.

La tabla 5 resume los resultados del Test de Kolb.

Tabla 5

Matriz resultados Test de Kolb

\begin{tabular}{|c|c|c|c|c|}
\hline \multicolumn{5}{|c|}{ Resultados Test de Kolb dirigido a colaboradores ICBF } \\
\hline \multirow[b]{2}{*}{ Colaborador } & \multicolumn{4}{|c|}{ Estilo de aprendizaje } \\
\hline & $\begin{array}{l}\text { Conver- } \\
\text { gente }\end{array}$ & $\begin{array}{l}\text { Diver- } \\
\text { gente }\end{array}$ & $\begin{array}{c}\text { Asimila- } \\
\text { dor }\end{array}$ & $\begin{array}{c}\text { Acomoda- } \\
\text { dor }\end{array}$ \\
\hline 1 & & & & $x$ \\
\hline 2 & & $\mathrm{X}$ & & \\
\hline 3 & & & $\mathrm{x}$ & \\
\hline 4 & & $\mathrm{X}$ & & \\
\hline 5 & & & & $\mathrm{x}$ \\
\hline 6 & & & $\mathrm{X}$ & \\
\hline 7 & & $\mathrm{X}$ & & \\
\hline 8 & & $\mathrm{X}$ & & \\
\hline 9 & & $\mathrm{X}$ & & \\
\hline 10 & & $\mathrm{x}$ & & \\
\hline Total & 0 & 6 & 2 & 2 \\
\hline Porcentajes & $0 \%$ & $60 \%$ & $20 \%$ & $20 \%$ \\
\hline
\end{tabular}

Fuente: Elaboración propia.

La distribución de los porcentajes, según los estilos de aprendizaje obtenidos, se aprecian en la figura 7.

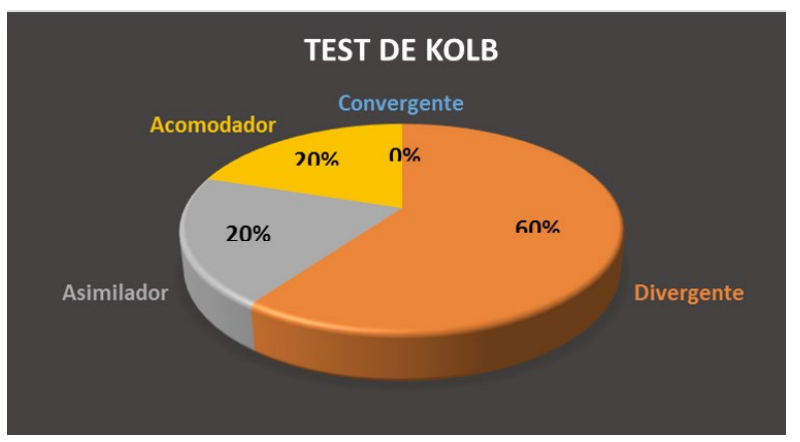

Figura 7. Resultados Test de Kolb. Fuente: Elaboración propia. 
Ambiente Virtual de Aprendizaje para la Capacitación Tipo Inducción, Sobre Contenidos Estratégicos y Misionales Dirigido a Colaboradores del Instituto Colombiano de Bienestar Familiar (ICBF)

A partir de los porcentajes calculados, se obtuvieron las características por estilo de aprendizaje (CCA, 2012), que se describen en la tabla 6 .

Tabla 6

Características y dominio de cada estilo de aprendizaje

\begin{tabular}{|c|c|c|}
\hline $\begin{array}{l}\text { Estilo de } \\
\text { aprendizaje }\end{array}$ & Dominio & Caracterizado porque \\
\hline \multirow[t]{2}{*}{ Divergente } & $60 \%$ & $\begin{array}{l}\text { Se basan en experiencias concretas } \\
\text { (EC), aunque su punto fuerte es la ca- } \\
\text { pacidad imaginativa. Por lo tanto, son } \\
\text { excelentes productores de diferentes } \\
\text { ideas. También poseen una observación } \\
\text { reflexiva (OR), proporcionando herra- } \\
\text { mientas para el pensamiento y planea- } \\
\text { ción. }\end{array}$ \\
\hline & & $\begin{array}{l}\text { Características: Sensorial o kinestési- } \\
\text { cos (toman decisiones en función de lo } \\
\text { que sienten), experimentales, flexibles, } \\
\text { creativos y tienen iniciativa. }\end{array}$ \\
\hline \multirow[t]{2}{*}{ Asimilador } & $20 \%$ & $\begin{array}{l}\text { Son lógicos al organizar información, } \\
\text { combinando la conceptualización abs- } \\
\text { tracta (CA) y la observación reflexiva } \\
\text { (OR). Se encuentran más interesados } \\
\text { en las ideas abstractas y conceptos, } \\
\text { prevaleciendo lo lógico frente a lo prác- } \\
\text { tico. }\end{array}$ \\
\hline & & $\begin{array}{l}\text { Características: En ellos predominan } \\
\text { las carreras de tipo científico o analíti- } \\
\text { co. Son metódicos, lógicos, racionales y } \\
\text { sistemáticos. }\end{array}$ \\
\hline
\end{tabular}

Acomodador $20 \% \quad$ Aprenden de la experiencia concreta (EC), con iniciativa para participar en cosas nuevas y la combinan con la experiencia activa (EA) para la ejecución de planes. Son fácilmente adaptables a nuevas circunstancias específicas.

Características: Arriesgados, poco pacientes, emocionales, intuitivos, imaginativos y observadores de detalles específicos.

Convergente $\% \quad$ Aprenden a través de la solución de problemas, buscan la práctica a través de las ideas. Toman decisiones a través de los resultados encontrados para encontrar o definir una sola rama o resultado como conclusión.

Características del convergente: Hábiles para interpretar la teoría a la práctica, excelentes para los puestos técnicos.

Fuente: CCA (2012).

De acuerdo a la clasificación anterior, se deduce que, dentro de la caracterización del estilo de aprendizaje aplicado a 10 colaboradores aleatorios del ICBF, predomina el tipo divergente a comparación del convergente, que no obtuvo resultados.
Se encontró que el $60 \%$ de la población estudiada (6 de 10 personas), se inclinan hacia el aprendizaje divergente, caracterizándose por aprender a partir de sensaciones, ser experimentales, flexibles y creativos. Dentro de esta caracterización, los individuos evidencian habilidades en consejería, administración del personal y como especialistas en desarrollo organizacional (Córdova, 2015). Estos resultados son importantes porque contar con colaboradores de estas características contribuye todavía más al crecimiento visional y misional del Instituto (García, 2016). Ahora bien, para determinar qué tipo de inteligencia poseen, fue necesario tener en cuenta el modelo de cuatro cuadrantes de Kolb (CCA, 2012), que se describe en la figura 8.

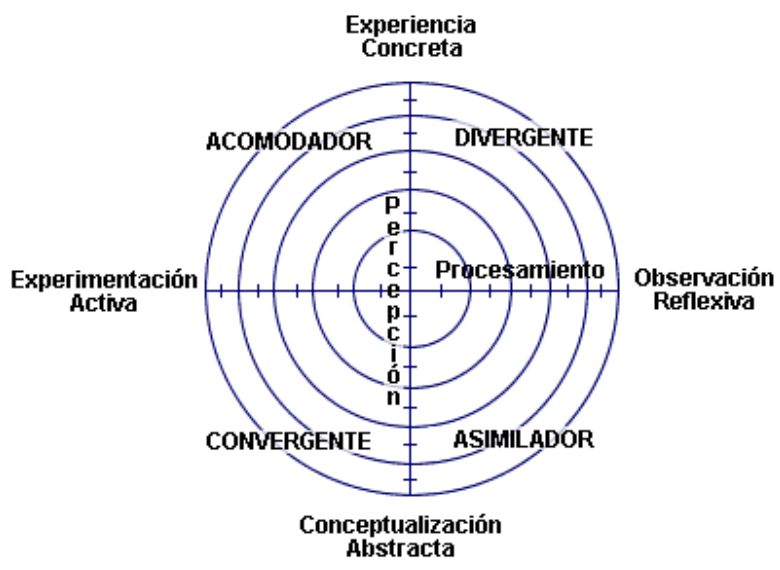

Figura 8. Modelo de cuatro cuadrantes de Kolb (CCA, 2012).

Como el estilo de aprendizaje divergente se ubica en medio de los cuadrantes Observación Reflexiva (OR) y Experiencia Concreta (EC), identificamos una combinación entre el tipo de aprendizaje reflexivo y el activo.

A partir de los resultados, para el estilo reflexivo se utilizan colores rosa, marrón y verde, mientras que para el estilo activo se emplean tonos amarillo, rojo y naranja. Esto obedece a que los colores predominantes coinciden dentro de los usados tanto en el AVA Introducción al ICBF, como para el OVA Misional Estratégico. A continuación, en la siguiente figura 9 se evidencia la aplicación de estos colores. 


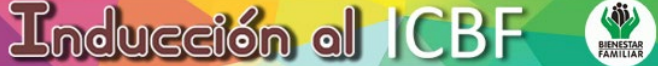

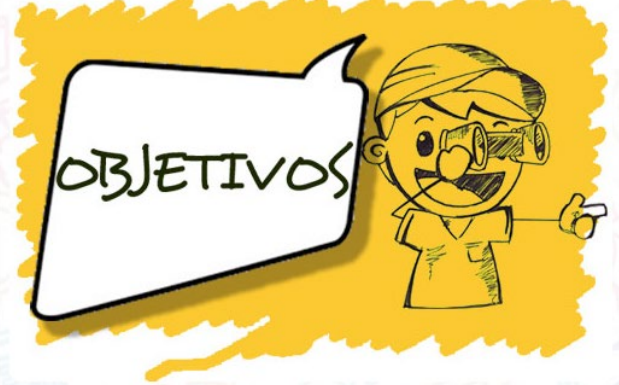

Figura 9. Aplicación tipo de colores según inteligencias múltiples.

Por otro lado, se tiene en cuenta la distribución de resultados de la encuesta de satisfacción 1 , mediante diagramas de torta, de manera inicial a las preguntas relacionadas con el desarrollo de contenido (figura 10), donde el $80 \%$ de los colaboradores opinaron que los contenidos están lógicamente organizados; el 90\% indicó que el lenguaje de los temas y módulos fue el adecuado y el 90\% indicó que la información es clara y precisa.

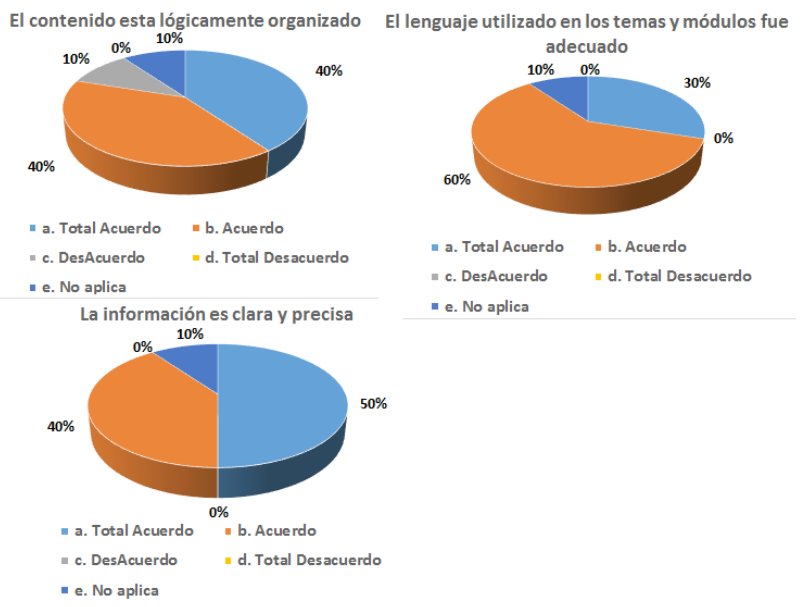

Figura 10. Resultados para las preguntas desarrollo de contenido.

De manera similar, más del 70\% de la población opinó que se cumplieron con los objetivos de las actividades, se verificaron los temas propuestos y que el manejo de la plataforma fue sencillo (figura 11).

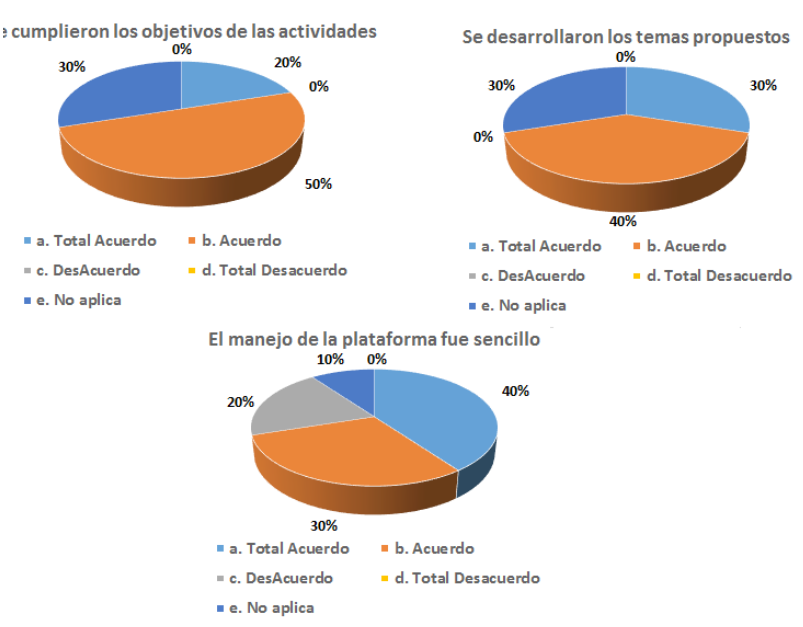

Figura 11. Resultados para las preguntas objetivos del curso

Para preguntas relacionadas con la resolución de inconvenientes durante el curso y el tiempo de respuesta frente a ellos (figura 12), prevaleció la respuesta no aplica, ya que la prueba era solo de navegabilidad más no de certificación de los contenidos. Por otro lado tan sólo el $10 \%$ opino que las inquietudes planteadas no fueron resueltas satisfactoriamente y el $80 \%$ de los colaboradores ICBF opinaron que el curso cumplió con sus expectativas.

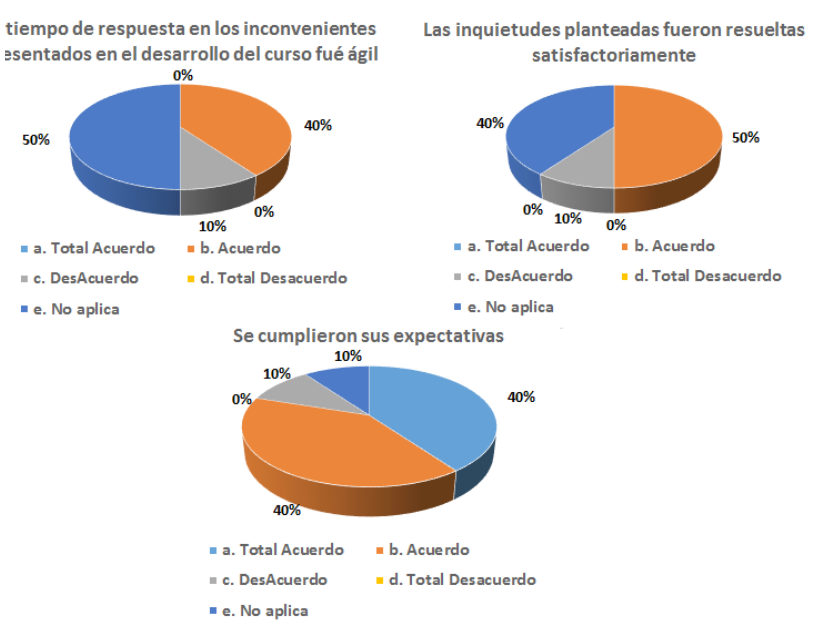

Figura 12. Resultados para las preguntas tiempo de respuesta.

Respecto a los contenidos, actividades y evaluación (figura 13), el 90\% opinó que el apoyo tecnológico fue pertinente en el aprendizaje de contenidos, transferible a la práctica profesional y coherente, pertinente y actual con los objetivos propuestos. 
Ambiente Virtual de Aprendizaje para la Capacitación Tipo Inducción, Sobre Contenidos Estratégicos y Misionales Dirigido a Colaboradores del Instituto Colombiano de Bienestar Familiar (ICBF)

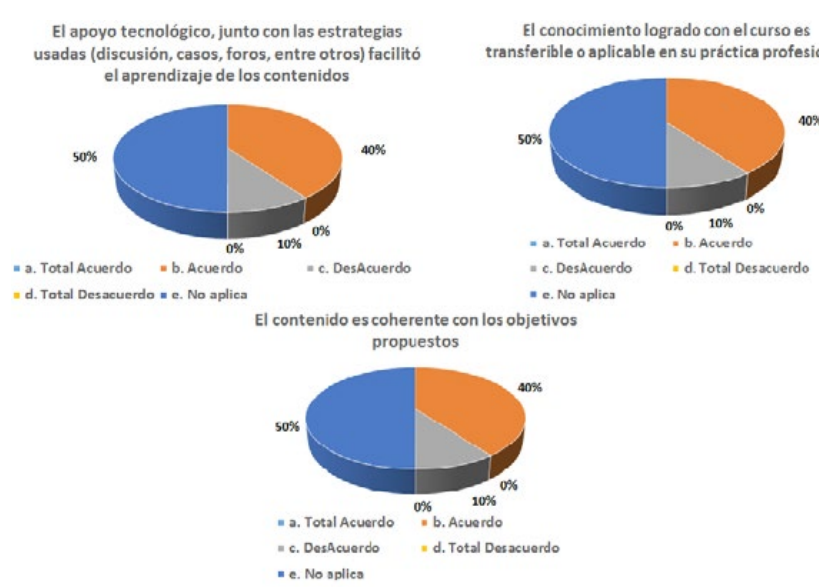

Figura 13. Resultados para las preguntas referidas a contenidos, actividades y evaluación.

En cuanto a las fechas de entrega de las actividades (figura 14), más del 70\% indicó que estas fueron claras y el $90 \%$ estuvo de acuerdo que se informó con claridad los objetivos.

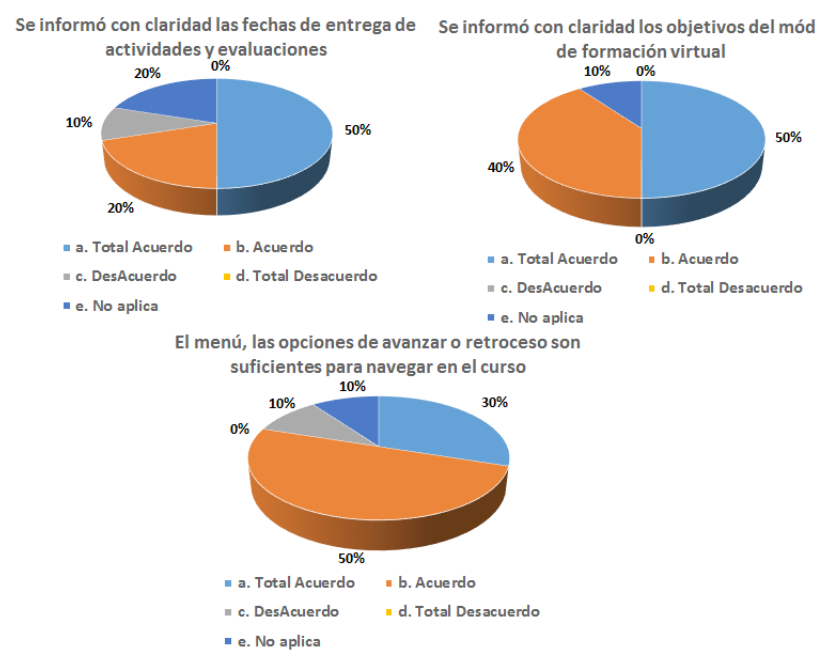

Figura 14. Resultados para las preguntas tiempo de entrega e interacción gráfica.

Por otro lado, para la interacción gráfica (figura 15 ) el $80 \%$ de los usuarios opinaron que las ilustraciones fueron apropiadas, al igual que las interacciones.

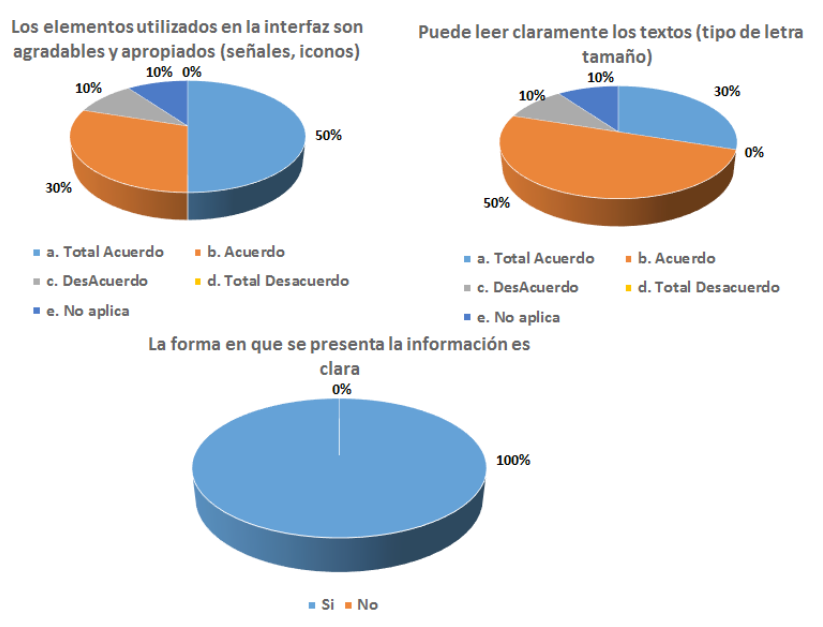

Figura 15. Resultados para las preguntas representación gráfica (parte 2).

Para la Encuesta 2: Evaluación ambiente virtual de aprendizaje, que tiene en cuenta la relevancia del AVA (figura 16), los usuarios indicaron que casi siempre los temas fueron interesantes, importantes y estaban relacionados con su práctica.

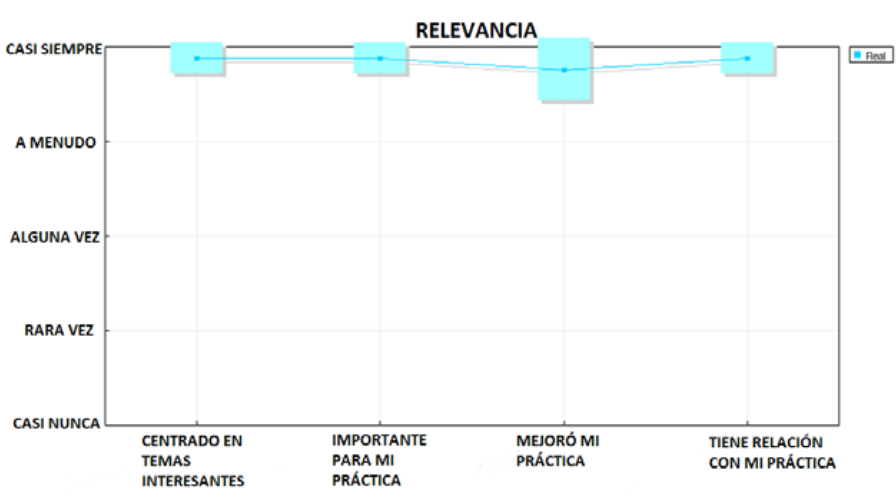

Figura 16. Resultados para la relevancia del AVA.

Por otro lado, en el pensamiento reflexivo (figura 17), los colaboradores a menudo fueron críticos en el aprendizaje, con sus ideas, con otros estudiantes y con lo que leen.

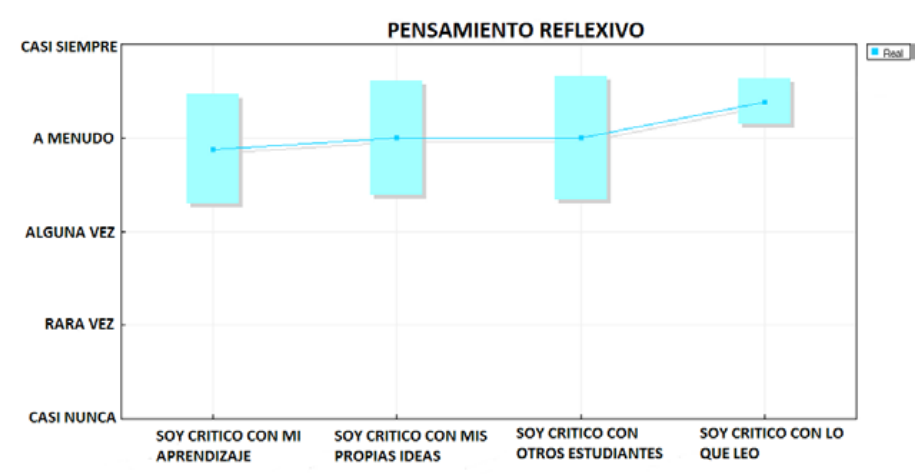

Figura 17. Resultados para el pensamiento reflexivo. 
Dentro de las relaciones con el tutor (figura 18), cabe resaltar, según ellos ,que a menudo su tutor estimuló la reflexión, lo animó, casi siempre ejemplificó sus disertaciones y ejemplificó la autoreflexión.

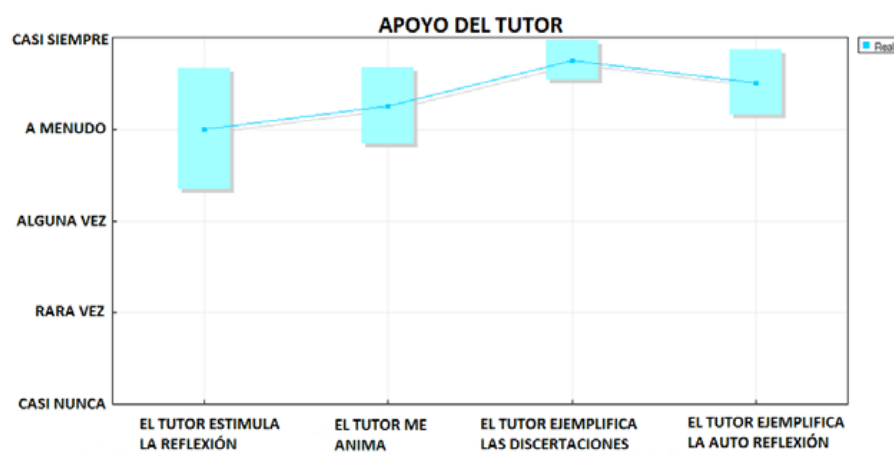

Figura 18. Resultados relaciones con el tutor.

Además, se evidenció que, a menudo, los estudiantes se animan con el apoyo de los compañeros, se elogian entre ellos, los valoran y empatizan (figura 19).

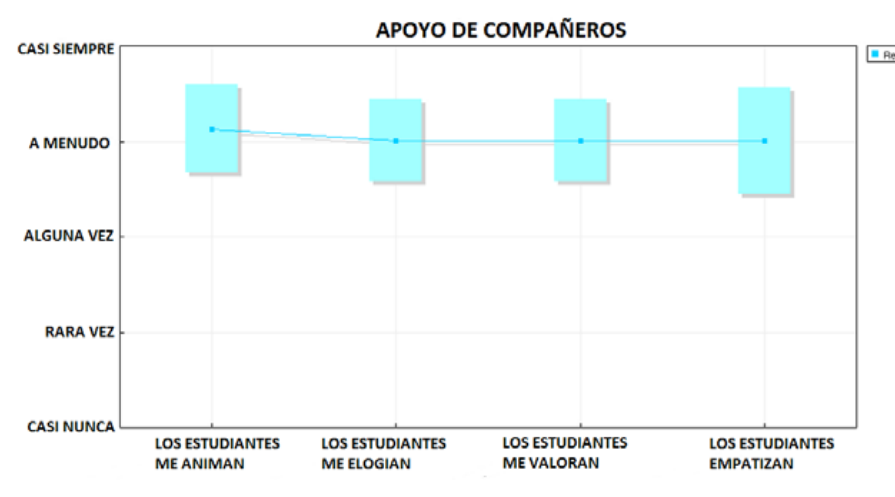

Figura 19. Resultado apoyo de compañeros.

En cuanto a la interpretación (figura 20), los colaboradores ICBF evaluados, a menudo entienden a otros estudiantes; otros estudiantes los entienden y casi siempre entienden al tutor y, a su vez, el tutor los comprende.

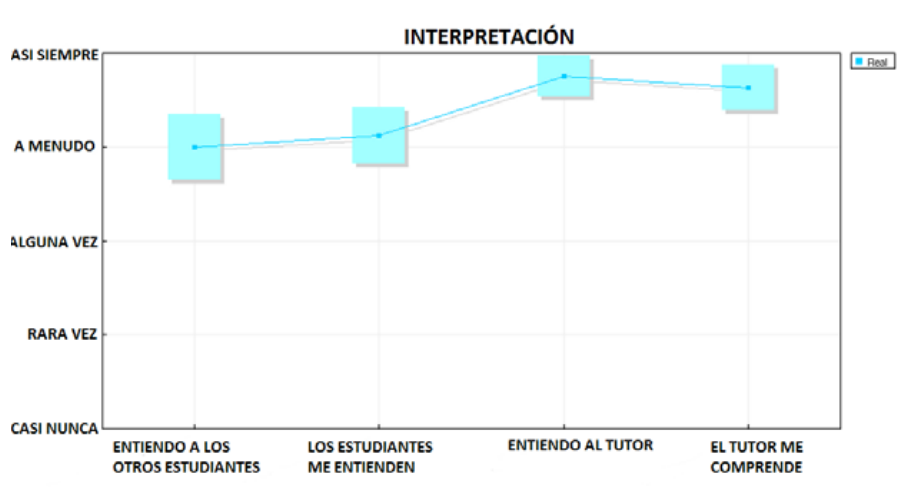

Figura 20. Resultados para la interpretación.

\section{Discusión, Conclusiones Y RECOMENDACIONES}

Las estrategias planteadas en la investigación de Huertas en Colombia indican la necesidad de plantear contenidos o enunciados expresados en lenguaje natural o simbólico, imágenes, dibujos, expresiones y gráficos (Huertas, 2015). Ese hallazgo fue también relevante en la presente investigación, ya que no sólo incrementó el nivel motivacional de los colaboradores por su interacción con el ambiente de aprendizaje mediante animaciones, sino que también ayudó a conocer cualitativamente su opinión acerca de los contenidos y el diseño del ambiente.

En consecuencia, se puede concluir que:

1. El diseño de un AVA permitió consolidar una cultura enfocada en el desarrollo de un trabajo sistémico. Generó impacto social y actuó de manera coherente con los objetivos y valores institucionales del ICBF; asimismo, favorece el cumplimiento de la misión institucional.

2. Mediante estrategias tecnológicas, constantemente, en el instituto se busca fortalecer la capacidad organizacional mediante intervenciones focalizadas (Nacional - Regional - Zonal) que permitan potenciar la excelencia y el desarrollo humano de los colaboradores para el logro de cada uno de los objetivos estratégicos, formándose colaboradores competentes, cualificados y alineados con la estrategia organizacional e integrando modalidades de 
aprendizaje (presencial, virtual y semipresencial).

3. Con el tiempo, si bajo la misma AVA se ingresan más contenidos para los módulos, con el objeto de capacitar al colaborador ICBF, será necesario documentar no sólo su construcción (Guion técnico), sino también buscar que los estudiantes se certifiquen en los diferentes módulos, fuera del estratégico y misional.

4. A su vez, es importante que, mientras se incremente el número de temas, estos se vean con el tiempo reflejados en la rúbrica, contenidos y ruta de ingreso para una óptima navegabilidad del AVA y OVA's. Así se acomodarán los recursos en una plataforma propia del ICBF y no bajo la administración de otra institución.

5. Por otro lado, debe existir una navegabilidad intuitiva, adicional a textos legibles, y recursos digitales apropiados para temas misionales y estratégicos del ICBF.

Sin duda, la incorporación de ambientes de aprendizaje web en las dinámicas corporativas sociales promueve el interés de los colaboradores mediante actividades de capacitación misional y visional.

De lo anterior se desprenden las siguientes recomendaciones:

1. Una de las recomendaciones a tener en cuenta es la necesidad de contar con mecanismos de soporte técnico para los roles de maestro y usuario, en cuanto a políticas de acceso a la plataforma y, también, de reserva de los contenidos, en caso de que la plataforma llegue a fallar; es decir, mediante algún ataque informático que implique la pérdida de la información.

2. Otro aspecto a resaltar es que los colaboradores que reciban la capacitación estratégica y misional bajo la modalidad virtual, conozcan de antemano el uso y manejo de herramientas ofimáticas e informáticas.

Entre las limitaciones a superar está la disponibilidad de herramientas computacionales en cada regional, pues resulta relevante considerar que la plataforma a utilizar (en este estudio la plataforma Kenta de prueba bajo Moodle LMS) no debe presentar problemas para activar y matricular usuarios en el ambiente virtual. Al poner al servicio del público este tipo de ambientes virtuales, es necesario que existan herramientas computacionales en cada regional con soporte de internet para el ingreso y la continuidad del curso.

\section{AGRADECIMIENTOS}

A la Universidad Pedagógica Nacional - UPN y su grupo de investigación KENTA de la Facultad de Ciencia y Tecnología, por permitir la orientación de tesis de posgrado en la Especialización en Tecnologías de la Información Aplicadas a la Educación y en la Maestría en Tecnologías de la Información Aplicadas a la Educación.

\section{REFERENCIAS BibliográficAs}

Córdova, F., Díaz, M., Cifuentes, F., Cañete, L., \& Palominos, F. (2015). Identifying problem solving strategies for learning styles in engineering students subjected to intelligence test and EEG monitoring. Procedia Computer Science, 55, 18-27. Recuperado de: https://doi.org/10.1016/j. procs.2015.07.003

Beltrán, J. (2016). La educación virtual en Colombia: la implementación de las TIC en la educación superior. Academia y Virtualidad, 4(1), 6-21. Recuperado de: https:/doi. org/10.1016/j.procs.2015.07.003

Casas, L. (2016). Tecnologías de comunicación e información en educación: Referentes para el análisis de entornos virtuales de enseñanza y aprendizaje. Revistas de Investigación, 33 (68).

Centros Comunitarios de Aprendizaje CCA (2012). Modelo de David Kolb, aprendizaje basado en experiencias.

García, M. (2016). Correlación inherente de los estilos del aprendizaje y las estrategias de enseńanza-aprendizaje. Revista Iberoamericana de Producción Académica y Gestión Educativa.

Huertas, O. (2015). Una interpretación semántica de la lectura y comprensión de los problemas de matemáticas en las pruebas externas nacionales en el grado quinto. Tesis de maestría inédita). Universidad Nacional de Colombia. Recuperado a partir de http://www.bdigital.unal.edu.co/48880/

Instituto Colombiano de Bienestar Familiar, De la Fuente, C. (2011). Contexto. Plan y objetivos estratégicos. Bogotá D.C. 
ICBF. (2013). Asistencia Técnica, Divulgación y Capacitación a Servidores Públicos para la Administración del Estado. DAPS, 2.

ICBF. (2014) ¿Qué es el ICBF?. Recuperado de: http:// www.icbf.gov.co/portal/page/portal/PortalICBF/EiInstituto

Medina, J., Martín, Y. y Beltrán, J. (2016). La responsabilidad social empresarial (RSE) en las organizaciones públicas: estudio de caso "Ecopetrol". Academia y Virtualidad, 6(1), 92-113. 